\title{
Anatomische Landmarken operativer Zugangswege an der Hand
}

\author{
Rosemarie Fröber, Jens Geiling
}

\section{Zusammenfassung}

Die Funktionen der Hand sind wie in keiner anderen Region des Körpers abhängig von der Intaktheit der anatomischen Strukturen. Der Weichteilmantel der Hand ist außerordentlich komplex und vielgestaltig. Er unterliegt ganz unterschiedlichen funktionellen Beanspruchungen, die sich in einer optimalen Anpassung der einzelnen anatomischen Strukturen widerspiegeln. Daher ist es wichtig, sich vor jedem operativen Eingriff der jeweils vorliegenden funktionel- len und anatomischen Gegebenheiten genau bewusst zu sein. Der Operateur muß darüber hinaus auf Überraschungen gefasst sein, da sich gerade die Hand durch eine Vielzahl anatomischer Varianten auszeichnet. Letztlich ist das funktionelle Operationsergebnis in hohem Maße davon abhängig, wie schonend der Zugangsweg durch den Weichteilmantel war. Daher soll nachfolgend an ausgewählte anatomische Landmarken erinnert werden, denen der Operateur auf dem Weg zum Skelett der Hand und des handgelenksnahen Unterarmes begegnet.

\section{Einleitung}

Die operativen Zugangswege der Hand führen durch einen sehr komplexen Weichteilmantel, in dem anatomische Strukturen höchster funktioneller Wertigkeit auf engstem Raum konzentriert und mit dem Skelett eng verwoben sind. In keiner anderen Region des Körpers ist die Funktion so stark auf einen unversehrten Weichteilmantel angewiesen wie an der Hand. Um beim Operieren den iatrogenen Schaden so klein wie möglich zu halten, ist es notwendig, maximal schonende Zugangswege zu wählen. Die zentrale Frage bei der Planung eines operativen Eingriffes ist daher: „Können die Weichteile das vertragen, was man ihnen zumutet?".

\section{Palmare Zugangswege zum distalen Radius und zum Karpalkanal}

Bei der Inspektion der Palmarseite sieht man die gewöhnlich als Grenze zwischen

OP-JOURNAL 2001; 17: 222-227

(c) Georg Thieme Verlag Stuttgart · New York
Unterarm und Hand angegebene starke Hautfurche (Rascetta), die aber tatsächlich schon im Bereich der Handwurzelknochen liegt. An deren ulnarem Ende tastet man das Os pisiforme sowie die daran inserierende Sehne des M. flexor carpi ulnaris (Abb.1 u. 2). In der Mitte der Beugefalte lassen sich zwei weitere Sehnenstrukturen identifizieren, die dem M. palmaris longus (kann gelegentlich auch fehlen) und dem radial davon gelegenen $M$. flexor carpi radialis zuzuordnen sind. Letztere ist Leitstruktur beim palmaren Zugang zum distalen Radius. Hier trifft man im Subkutangewebe auf feine sensible Endverzweigungen des N. musculocutaneus und des $N$. radialis. Um diese beim weiteren Vordringen zu schonen, sollten sie nach radial verlagert werden. Trennt man die Faszie über der Flexor-carpi-radialis-Sehne auf, so umgeht man die Gefahr, den ulnar benachbart verlaufenden $R$. palmaris des $N$. medianus zu verletzen. Dieser Hautast des $N$. medianus separiert sich bereits ca. $5 \mathrm{~cm}$ proximal der Rascetta, tritt in die Faszie ein und verläuft zwischen zwei Blättern der Faszien bis er diese am ulnaren Umfang des Thenar durchbricht und seine wichtigen sensiblen Endverzweigungen für den Handteller ausbildet.

\section{Abkürzungsverzeichnis zu den Abbildungen}

(1) M. palmaris longus

(2) M. palmaris brevis

(3) M. flexor carpi ulnaris

(4) M. flexor carpi radialis

(5) M. flexor pollicis longus

(6) $\mathrm{Mm}$. flexores digitorum superficialis et profundus

(7) M. adductor pollicis

(8) Mm. abductor pollicis longus et extensor pollicis brevis

(9) M. extensor pollicis longus

(10) M. interosseus primus

(11) Mm. lumbricales et interossei

(12) M. abductor pollicis brevis

(13) Os pisiforme

(14) Processus styloideus radii

(15) Processus styloideus ulnae

(16) Palmaraponeurose der Hohlhand

(17) Lig. carpi palmare

(18) Retinaculum flexorum

(19) Retinaculum extensorum

(20) Connexus intertendinei

(21) Strecksehne

(22) Sehnenscheide

(23) Streckaponeurose der Finger

(24) Lig. retinaculare obliquum Landsmeer

(25) Clelandsche Hautbänder

(26) Graysonsche Hautbänder

(27) R. cutaneus des N. musculocutaneus

(28) R. superficialis des N. radialis

(29) R. palmaris des N. medianus

(30) R. thenaris des N. medianus

(31) R. superficialis des N. ulnaris

(32) R. profundus des N. ulnaris

(33) A. et N. digitalis dorsalis; dorsale Gefäß-Nervenstraße der Finger

(34) A. et N. digitalis palmaris proprius; palmare Gefäß-Nervenstraße der Finger

(35) A. ulnaris

(36) A. radialis

(37) R. superficialis der A. radialis

(38) R. carpalis dorsalis

(39) A. metacarpalis dorsalis I 


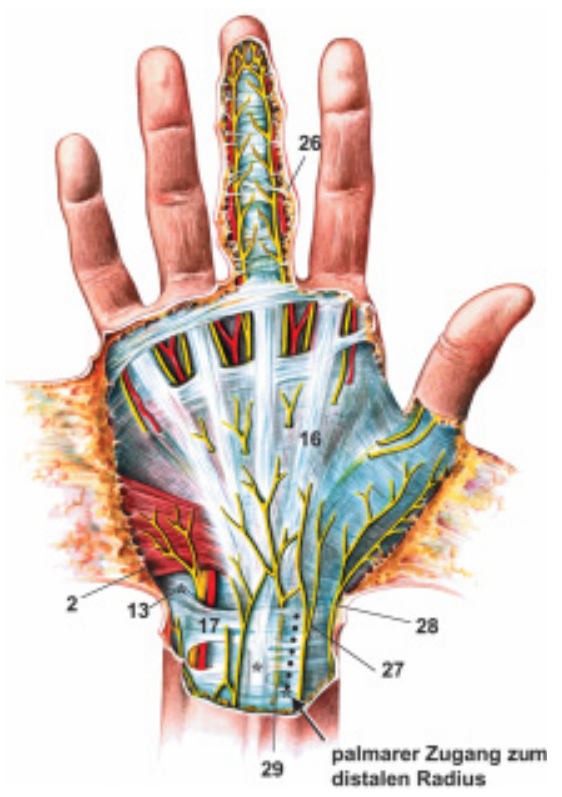

Abb.1. Die extrafaszialen und palpierbaren* Strukturen der Hohlhand

Der für die sensible Innervation des Handtellers wichtigste Hautnerv R. palmaris $n$. mediani sollte beim palmaren Zugang zum distalen Radius geschont werden.

Unter der Faszie trifft man ulnar der sehnigen Leitstruktur (Flexor-carpi-radialis-Sehne) auf den $N$. medianus, der hier eine mittlere Breite von $6 \mathrm{~mm}$ aufweist. Gemeinsam mit den Sehnen der langen Fingerbeuger verläuft er zum Karpalkanal. Die Flexor-carpi-radialis-Sehne gelangt in einer eigenen, vom Canalis carpi separierten Gleitbahn zur Handwurzel und besitzt radialseitig engen Kontakt zur A. radialis. Letztere entlässt einen sehr variablen $R$. superficialis, der die Thenarmuskulatur versorgt und sich jenseits davon in etwa einem Drittel der Fälle an der Ausbildung des oberflächlichen Hohlhandbogens (Arcus palmaris superficialis) beteiligt. Unmittelbar distal des tastbaren Knochenvorsprunges am Radius, dem Processus styloideus radii, unterkreuzt die Arterie zwei zum Daumen verlaufende Sehnen (Mm. abductor pollicis longus et extensor pollicis brevis) und gelangt so auf den Handrücken in die Tabatiere.

Der $N$. medianus ist den benachbarten Beugesehnen in Dicke und Lage zum Verwechseln ähnlich.

Auf dem Wege zum Radius kommt unter der Flexor-carpi-radialis-Sehne die Sehne des M. flexor pollicis longus zu liegen, die von einem engen radialen Sehnenschei-

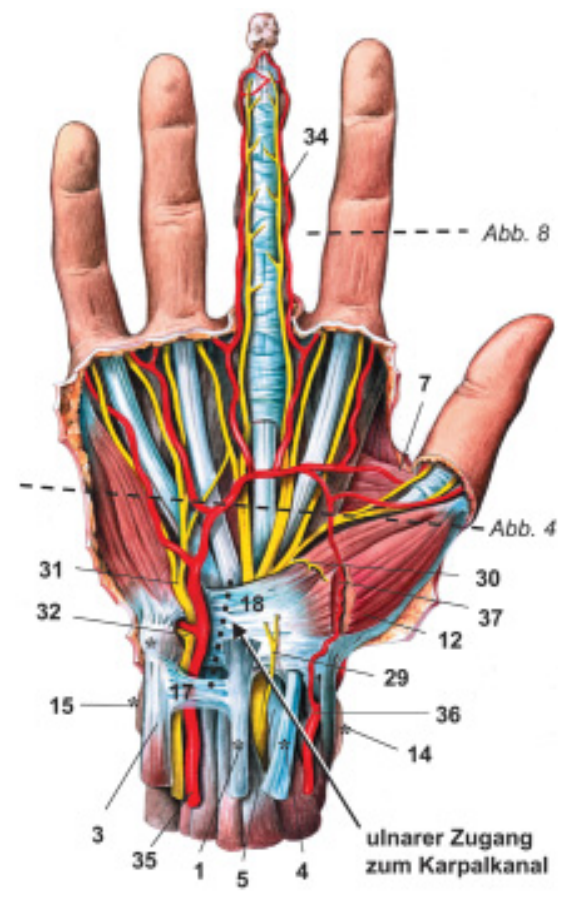

Abb. 2. Die subfaszialen und palpierbaren* Strukturen der Hohlhand

densack umhüllt wird. Unmittelbar ulnar davon verläuft der $N$. medianus mit den übrigen 8 Fingerbeugesehnen der $\mathrm{Mm}$. flexores digitorum superficialis et profun$d u s$, in lockerem Bindegewebe eingebettet. Dieses enthält sehr feine Gefäßnetze und gehört einem weiten ulnaren Sehnenscheidensack an. Um diese autochtonen Gefäße beim weiteren operativen Vordringen zu schonen, sollten Nerv und Sehnen en block nach ulnar verlagert werden. In der Tiefe erscheint der bei Frakturen häufig mitverletzte $M$. pronator quadratus. Wird dieser nahe seines radialen Ansatzes parallel zum Radiusschaft durchtrennt und abgeschoben, so liegt die distale Radiusmetaphyse frei.

Der $N$. medianus und die Beugesehnen der Finger sollten beim operativen Vordringen in ihrem ernährenden Bindegewebe belassen werden.

Der operative Zugang zur Entlastung des Karpalkanals, Canalis carpi, führt durch eine zweischichtige bindegewebige Abdeckung (Abb.1u.2). Die oberflächliche Schicht besteht aus der sich zur Palmaraponeurose verbreiternden Sehne des $M$. palmaris longus sowie dem Lig. carpi palmare, einer Verstärkung der oberflächlichen Faszie. Unmittelbar darunter trifft man auf das straffe Retinaculum flexorum, das den Karpalkanal verschließt. Zwischen beiden Bindegewebslagen verläuft der R. palmaris des N. medianus zur Hohl- hand. Der N. medianus selbst trifft unmittelbar unterhalb des Retinaculum flexorum im Karpalkanal ein. In $97 \%$ der Fälle liegt er den Beugesehnen der Finger auf und nimmt hier in der Regel eine radiale bis mediane Position ein, bevor er sich am Ausgang des Karpalkanals in seine Endäste aufspaltet (Abb.3a-c). Gelegentlich weist der Nerv aber auch Abbiegungen (33\%) oder eine ulnare Position (1,7\%) auf. In ca. 3\% der Fälle ist mit einer „hohen Teilung“ des Nerven zu rechnen (Abb.3d-f). Häufig ist diese mit einer persistierenden $A$. mediana antebrachii, einem akzessorischen $M$. lumbricalis oder abnormen Nervenverläufen kombiniert.

Der ulnare Zugangsweg zum Karpalkanal ist aus anatomischer Sicht mit den geringsten Risiken verbunden.

Unmittelbar radial des tastbaren Os pisiforme verbreitert sich der Raum zwischen Lig. carpi palmare und Retinaculum flexorum zum ulnaren Hohlhandkanal, „Loge de Gyon" (Abb.2). Dieser beherbergt die ulnare Gefäß-Nervenstraße in sehr konstanter topografischer Anordnung. Dem Canalis carpi eng benachbart verläuft die A. ulnaris, die schließlich in den oberflächlichen Hohlhandbogen (Arcus palmaris superficialis) übergeht. Ulnar wird die Arterie vom N. ulnaris begleitet. Dieser innerviert mit seinem sensiblen Endast, $R$. superficialis $n$. ulnaris, eineinhalb Finger palmar und bildet gelegentlich Anastomosen mit den sensiblen Endverzweigungen des $N$. medianus aus. Der motorische $R$. profundus $n$. ulnaris tritt gemeinsam mit einem Ast der Arterie in die Muskulatur des Hypothenar ein, um diese zu innervieren und schließlich in die tiefe Hohlhand zur intrinsischen Muskulatur bzw. zum M. adductor pollicis weiter vorzudringen.

Der Scheitel des oberflächlichen Hohlhandbogens ist nur 1-2 Querfinger vom Ausgang des Karpaltunnels entfernt und projiziert sich auf die Höhe der 1. Interdigitalfalte.

Unmittelbar unter dem Retinaculum flexorum verläuft der $N$. medianus mit seinen sensiblen Endverzweigungen für dreieinhalb Finger palmar. Der $R$. thenaris des $N$. medianus ist für die Innervation der Muskulatur des Daumenballens und damit für die funktionell bedeutsame Opponierbarkeit des Daumens zuständig. Dieser motorische Endast tritt in drei Viertel der Fälle erst distal des Canalis carpi in den Daumenballen ein, obwohl 


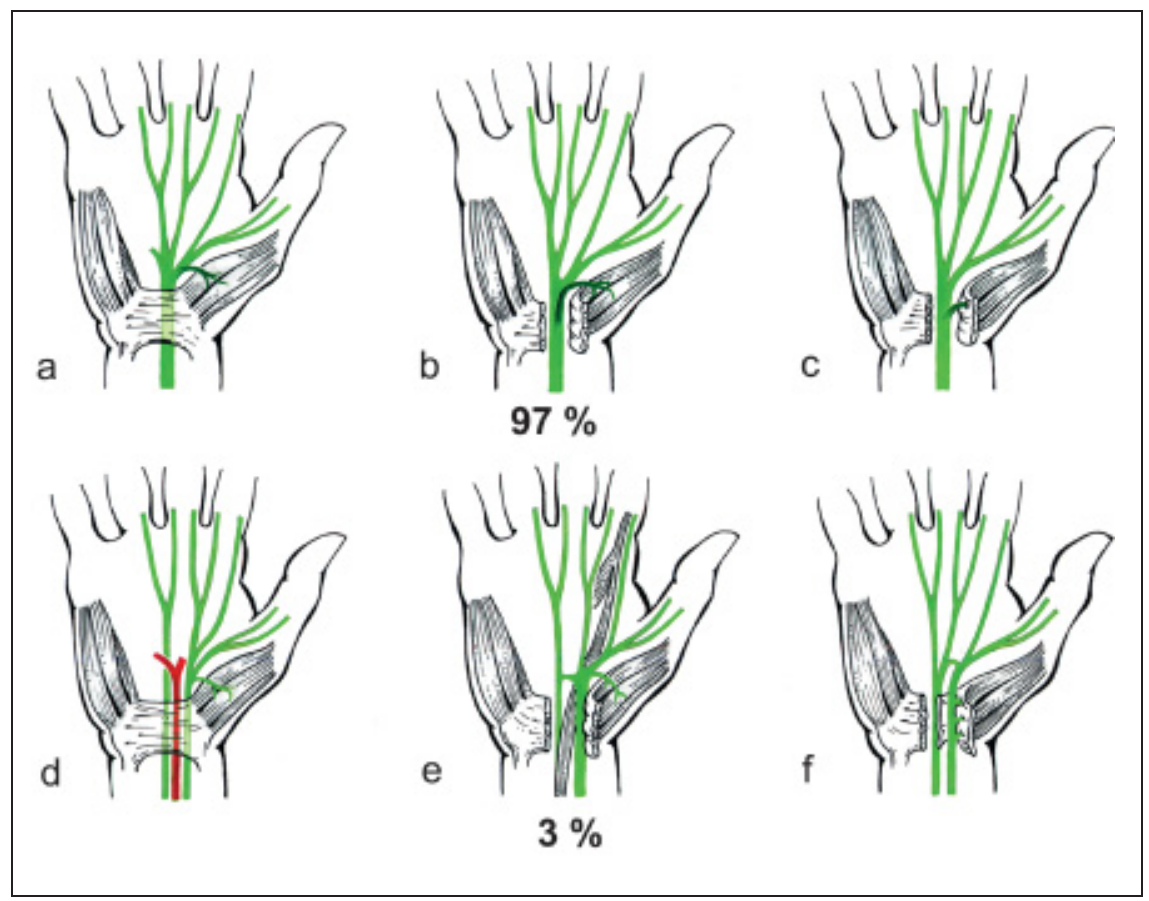

Abb.3. N. medianus: $a-c$ Verlaufsvarianten des R. thenaris und $d-f$ Variationen bei hoher Teilung

er den N. medianus in der Regel radialseitig bereits innerhalb des Karpalkanals verlässt (Abb. 3a, b, d,e e). Zu beachten ist die Variabilität des $R$. thenaris $n$. mediani, zu der u. a. sein transretinakulärer Eintritt in den Daumenballen und seine paarigen Verlaufsvarianten gehören (Abb.3c,f).

Die Verletzungsgefahr des motorischen Medianusastes zur Innervation des Daumenballens ist im ulnaren Abschnitt des Karpalkanals geringer als im radialen.

\section{Dorsale Zugangswege zum Handskelett}

Der Zugang zum Handskelett erfolgt in der Regel über den Handrücken. Dabei durchdringt man einen Weichteilmantel, dessen Dicke höchstens ein Viertel der des Handtellers beträgt (Abb.4). Im Unterschied zur Hohlhand, die aus einem architektonisch komplizierten Druckkammersystem besteht, sind die Weichteile des Handrückens in flachen, parallel zur Haut verlaufenden Lamellen angeordnet. Diese bilden eine oberflächliche und eine tiefe Faszienschicht sowie eine zwischen beiden befindliche Strecksehnenschicht. Da die Bewegungen der Hand mit extensiven Hautverschiebungen und ständigem Gleiten der Strecksehnen einhergehen, ist die Erhaltung einer ungestörten Gleitfähigkeit der Weichteile des Handrückens funktionell von großer Wichtig-

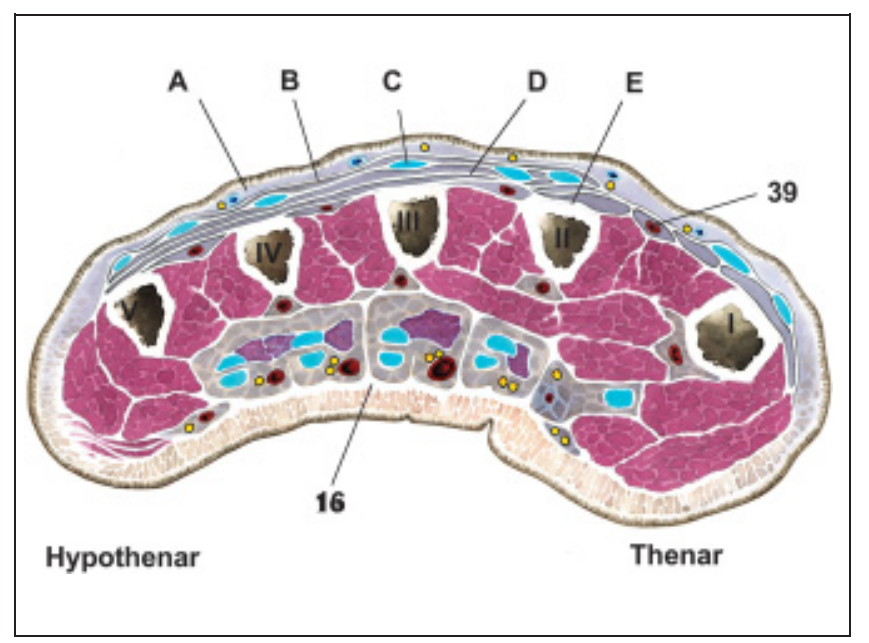

Abb.4. Querschnitt durch die Hand in Höhe der Schaftmitte der Mittelhandknochen (vergl. Abb. 2). Bindegewebslagen am Handrücken:

A Subkutanschicht mit Venengeflechten und sensiblen Endästen der $\mathrm{Nn}$. radialis et ulnaris B oberflächliche Faszie C Strecksehnenschicht keit. Sie beruht letztlich auf dem zwischen den Lamellen befindlichen lockeren Gleitgewebe sowie den Sehnenscheiden.

Bei der Präparation des Subkutangewebes trifft man auf ein variabel gestaltetes System von sehr dünnwandigen Handrückenvenen, die am Unterarm in die $V v$. basilica et cephalica einmünden. Diesen Venennetzen eng angeschlossen verlaufen die Lymphgefäße der Hand.

Großzügige Durchtrennungen von Venen sind zu vermeiden, da hier die gesamte Blut- bzw. Lymphdrainage erfolgt.

Unmittelbar unter den Venen verlaufen die sensiblen Endäste der Nn. ulnaris et radialis. Diese treten bereits am distalen Unterarm in die Subkutanschicht ein. Ca. $2 \mathrm{~cm}$ proximal des Processus styloideus ulnae perforiert der $R$. dorsalis $n$. ulnaris an der beugeseitigen Ulnakante die Faszie. Er verläuft zunächst palmar dieser knöchernen Landmarke und biegt unmittelbar distal davon zum Handrücken um. Hier treffen seine Endverzweigungen auf die des $N$. radialis, so dass sich die Innervationsgebiete in der Längsachse des Mittelfingers treffen. Der R. superficialis n. radialis perforiert die Faszie bereits ca. $7 \mathrm{~cm}$ proximal des Processus styloideus radii. Sein sensibles Aufzweigungsterminal befindet sich unmittelbar dorsal dieser radialen Knochenmarke und wird von der $V$. cephalica überquert. Die Endverzweigungen des Nerven passieren die sog. Tabatiere und verlaufen über deren ulnare Sehnenbegrenzung, die Sehne des M. extensor pollicis longus, zum Handrücken.

Die Hautnerven des Handrückens verlaufen bevorzugt in Längsrichtung; es ist aber auch mit bogenförmigen Querverbindungen zwischen den Ulnaris- und Radialisästen zu rechnen.

Führt der operative Zugang durch die Tabatiere, so müssen neben oberflächlichen Venen die sensiblen Endverzweigungen des $N$. radialis beachtet werden (Abb. 5). Nach Spaltung der Faszie findet man die $A$. radialis, die unmittelbar distal des Processus styloideus radii unter den Sehnen des ersten Sehnenscheidenfaches (Mm. abductor pollicis longus et extensor pollicis brevis) erscheint. Sie durchzieht die Tabatiere, verlässt diese unter der Sehne des $M$. extensor pollicis longus und gelangt zum ersten Intermetakarpalraum. Hier verschwindet die Arterie bajonettförmig abgeknickt zwischen den beiden Köpfen des M. interosseus primus, um 


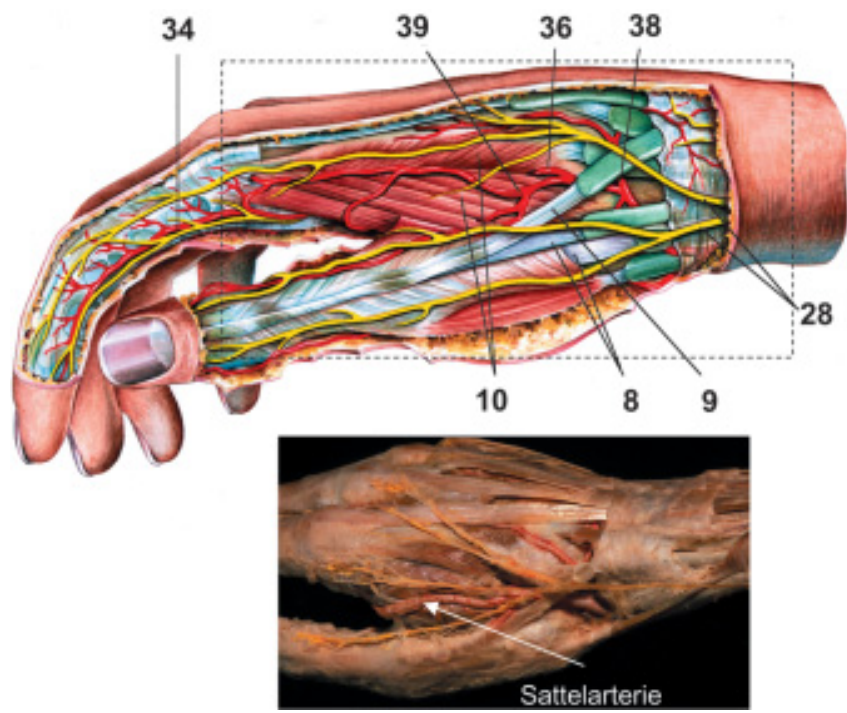

\section{D}

tiefe Faszie

E

Periost

Abb. 5. Topografische Anatomie der Tabatiere. Regelfall schematisch; Variante in situ. schließlich in der tiefen Hohlhand den Arcus palmaris profundus zu bilden. Vorher entlässt sie neben sehr variablen Ästen für den Daumen den $R$. carpalis dorsalis, der vor allem dann kräftig ausgebildet ist, wenn der tiefe Hohlhandbogen fehlt. $\mathrm{Zu}$ den Endästen der $A$. radialis gehört auch die A. metacarpalis dorsalis I, die sich an der Versorgung von Zeigefinger und Daumen beteiligt. In der ersten Interdigitalfalte kann sich diese mit den palmaren Zeigefinger- und Daumenarterien verbinden und eine direkt unter der Haut liegende „Sattelarterie“ ausbilden.

Sowohl beim Kahnbein- als auch beim Sattelgelenkzugang ist die topografische Anatomie der Tabatiere zu beachten.

Beim Zugang zu den Mittelhandknochen trifft man unter der oberflächlichen Faszie auf zwei- bis mehrfiedrige Strecksehnen, die fächerförmig nach distal auseinander streben, um in die Strecksehnenhaube der Finger einzustrahlen (Abb.6). Dabei projiziert sich der Sehnenverlauf zum Mittel- und zum Ringfinger auf den 3. und 4. Mittelhandknochen, während die Zeige- und Kleinfingersehnen den 2. und 4. Zwischenknochenraum kreuzen und daher nur die distalen Abschnitte der zugehörigen Mittelhandknochen tangieren. Die Strecksehnen der Langfinger sind durch eine dünne aponeurotische Platte verbunden, deren distaler Abschluss zu den Connexus intertendinei verstärkt ist. Im Anschluss an die Passage der Sehnenscheidenfächer unter dem Retinaculum extensorum werden die Strecksehnen von Sehnenscheiden begleitet, die z.T. in Höhe des Daumensat- telgelenkes enden, gelegentlich aber auch über die Mitte der Ossa metacarpalia hinausreichen. Distal der Sehnenscheiden liegen die Strecksehnen in einem bindegewebigen Gleitlager, dem Paratendineum, das jenseits der Sehnenscheiden feine ernährende Blutgefäße an jede Strecksehne heranführt.

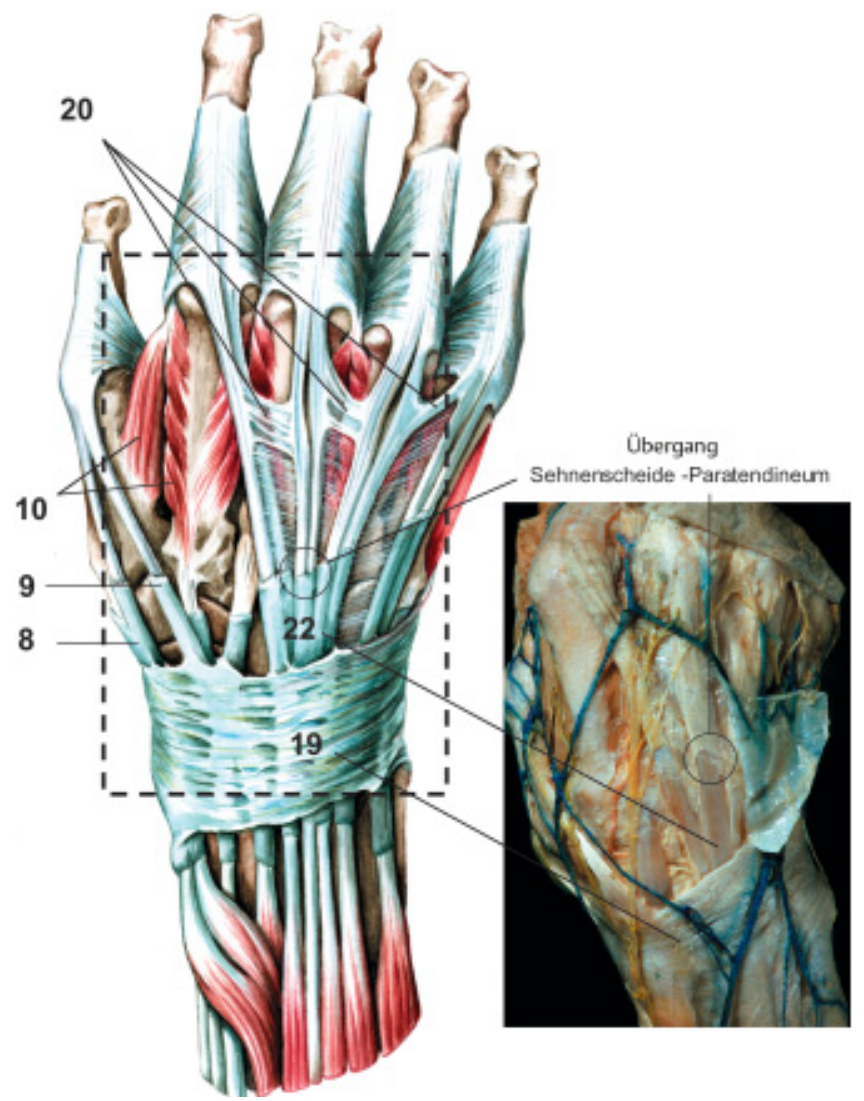

Beim operativen Zugang sind die einzelnen Bindegewebslagen des Handrückens schichtenweise zu inzidieren, ohne dass die Sehnen aus ihrer Umhüllung herausgelöst werden. Sehnen dürfen intraoperativ nicht glänzen!

Nach en-block-Verlagerung des Streckapparates trifft man auf die tiefe Faszie und schließlich auf das Periost. An Letzterem inserieren die $\mathrm{Mm}$. interossei, die versorgende Blutgefäße an den Knochen heranführen. Daher sollte diese Weichteilverankerung bei Inzision des Periostes an der Knochenhaut verbleiben und das Periost so sparsam wie möglich abgelöst werden.

Eine gefürchtete Komplikation der Knochenbruchheilung ist das Einsprossen der sehnenversorgenden Gefäße aus dem sehnigen Gleitlager in den Kallus, was zu Verklebungen und Ernährungsstörungen der Sehnen führt. Zur Vermeidung solcher adhäsionsfördernder Gefäßneubildungen sollte der Wundverschluss am Handrücken schichtenweise vorgenommen werden.

Beim Wundverschluss am Handrücken sollte zusammenfügt werden, was zusammen gehört! Das bedeutet: Periost

Abb. 6. Gleitlager der Strecksehnen des Handrückens schematisch und in situ 
und tiefe Faszie adaptieren; für Gleitschicht sorgen; intertendinöse Platte verschließen; für Gleitschicht sorgen; oberflächliche Faszie adaptieren und Haut verschließen.

\section{Zugangswege zum Skelett der Finger}

Der Zugang zum Skelett der Finger erfolgt in der Regel von dorsal oder lateral. Wichtigstes anatomisches Merkmal dieser Region ist der aponeurotische Sehnenstreckapparat, der äußerst innige und komplizierte Beziehungen zum Skelett aufweist. Verletzungen dieser Aponeurose und ihrer Verankerungen können daher zu erheblichen funktionellen Defiziten führen. An ihrer Unterseite ist die Streckaponeurose mit dem Periost durch ein lockeres bindegewebiges Gleitlager verbunden; über den Fingergelenken ist sie jedoch an den Gelenkkapseln fest verwachsen (Abb. 7). Im Bereich der Grundgelenke beginnt sie als „Strecksehnenhaube“, die sich allmählich verjüngt, bis sie terminal an Endglied und Nagelmatrix inseriert. Die Streckaponeurose wird nicht nur von den axial verlaufenden Strecksehnen gebildet, sondern darüber hinaus durch von palmar eintreffende kollaterale Zügel ergänzt. Hierbei handelt es sich um die Sehnen der intrinsischen Muskulatur (Mm. lumbricales et interossei). Im Bereich der Mittel-Gelenke sind darüber hinaus retinakuläre Bänder (Landsmeer) des Fingerskeletts in die Streckaponeurose integriert. Die Intaktheit all dieser Zügel ist für das Gleichgewicht der am Beugemechanismus beteiligten Muskeln von außerordentlich großer Bedeutung.

Die Wahl eines operativen Zuganges zum Skelett der Finger sollte unter Berücksichtigung der funktionellen Anatomie des Streckapparates erfolgen.

Beim Zugang von lateral sind die GefäßNervenstraßen der Finger gefährdet. Hier muss man die feinen dorsalen Bündel, die sich bereits an den Grund- und Mittelphalangen erschöpfen, von den Hauptversorgern der Finger unterscheiden.

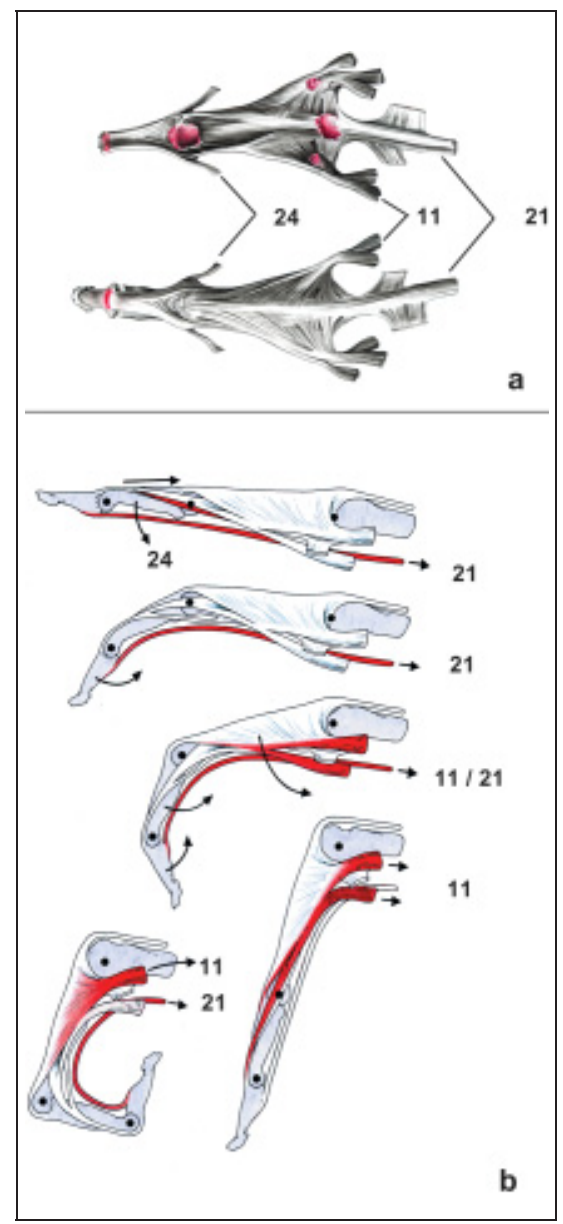

Abb. 7. Funktionelle Anatomie der Streckaponeurose am Finger.

a: Ansicht von palmar und dorsal

b: Beugemechanismus (modifiziert nach Tubina 1981).

Letztere stammen aus der Hohlhand und beinhalten neben Endverzweigungen des $N$. medianus und des $N$. ulnaris die großkalibrigen Aa. digitales palmares proprii. In Höhe der Phalangenbasen werden diese Versorgungsstraßen durch sog. „Hautbänder“ palmar (Grayson) und dorsal (Cleland) gezügelt (Abb.5 u. 8). Diese ligamentären Strukturen, die in ihrer Ausprägung großen individuellen Schwankungen unterliegen, verklammern die Haut an den tiefer gelegenen

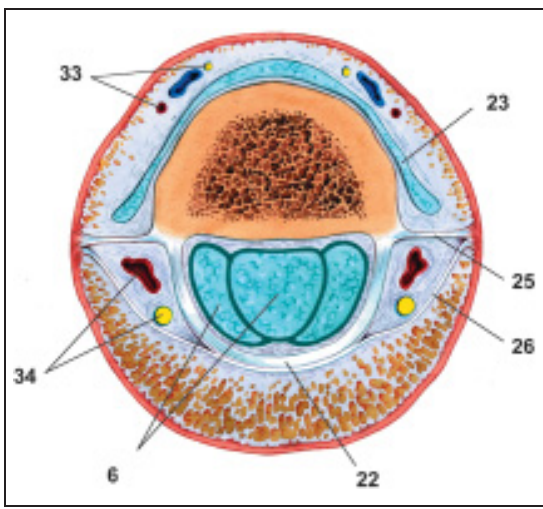

Abb. 8. Querschnitt durch den Zeigefinger in Höhe der distalen Grundphalanx (vergl. Abb. 2).

Bindegewebe- und Knochenstrukturen. Damit wird vor allem an der palmaren Seite eine übermäßige Verschiebbarkeit der Haut verhindert und das Greifvermögen verbessert. Die Gefäß-Nervenstraßen der Finger werden durch diese Hautbänder im Bereich der Phalangenbasen bzw. der lateral auslaufenden Hautbeugefalten fixiert.

Erfolgt der operative Zugang dorsal der Hautbeugefalten, so bleibt die Innervation und Versorgung der Finger unberührt.

\section{Literatur}

1 Schmidt H-M, Lanz U. Chirurgische Anatomie der Hand. Stuttgart: Hippokrates 1992

2 Pernkopf. Atlas der topographischen und angewandten Anatomie des Menschen. Hrsg. W. Platzer. München, Wien, Baltimore: Urban \& Schwarzenberg 1989

${ }^{3}$ Lanz-Wachsmuth. Praktische Anatomie. Berlin: Springer 1938

\section{Dr. med. Rosemarie Fröber}

\section{Oberärztin}

Institut für Anatomie I,

Medizinische Fakultät

Friedrich-Schiller-Universität Jena

D-07740 Jena 